\title{
Global uniqueness for a coefficient inverse problem for the non-stationary transport equation via Carleman estimate
}

\author{
Michael V. Klibanov and Sergey E. Pamyatnykh \\ Department of Mathematics and Statistics, \\ The University of North Carolina at Charlotte, Charlotte, NC 28223, U.S.A. \\ E-mails: mklibanv@email.uncc.edu, spamyatn@mail.ru
}

\begin{abstract}
A coefficient inverse problem for the non-stationary single-speed transport equation for $t \in(0, T)$ with the lateral boundary data and initial condition at $t=0$ is considered. Global uniqueness result is obtained via the method of Carleman estimates.

\section{Introduction}

The transport equation is used to model a variety of processes of particle transport, such as neutron diffusion, scattering of light in the atmosphere, propagation of $\gamma$-rays in scattering media, etc. (see, e.g., the book of Case and Zweifel [6]). Coefficient inverse problems (CIPs) for the transport equation are the problems of determining of the absorption coefficient, angular density of sources or scattering indicatrix. from an extra boundary data. They have a variety of applications in optical tomography, theory of nuclear reactors, etc. (see, e.g., the book of Anikonov, Kovtanyuk and Prokhorov [1] and references therein). This paper addresses the question of the global uniqueness for a CIP for the non-stationary single-speed transport equation with the extra lateral boundary data. Stability, uniqueness and existence results and references to such results for CIPs for the stationary transport equation can be found, e. e.g., in [1] and in the book of Romanov [25]. Uniqueness and existence results for CIPs for the non-stationary transport equation were obtained in the works of Prilepko and Ivankov [22], [23] and [24]. The results of [22] and [23] were obtained for special forms of the unknown coefficient using the overdetermination at a point. Also, uniqueness and existence results were obtained for an inverse problem, where complete lateral boundary data is not present but both initial and final conditions (at $t=T$ ) are given; see [24]. For some recent publications on overdetermined inverse problems for the transport equation see Tamasan [27] and Stefanov [26]. A derivation of the transport equation for the non-stationary case can be found, for example, in [6].

The proof of the main result of this paper is based on a Carleman estimate, obtained by Klibanov and Pamyatnykh [17]. Traditionally, Carleman estimates have been used for proofs of stability and uniqueness results for non-standard Cauchy problems for PDEs. They were first introduced by Carleman in 1939 [5], also see, e.g., books of Hörmander [7], Klibanov and Timonov [19] and Lavrent'ev, Romanov and Shishatskii [21]. Bukhgeim and Klibanov [4], [13] have introduced the tool of Carleman estimates in the field of CIPs for proofs of global uniqueness and stability results for CIPs, also, see Klibanov [14], [15], and Klibanov and Timonov [19], [20]. This method works for CIPs with single measurement data for the time dependent Partial Differential Equations (PDEs), as long as the initial condition is not vanishing and the Carleman estimate holds for the corresponding differential operator. Recently, Klibanov and Timonov have extended the original idea of [4] and
\end{abstract}




\section{Report Documentation Page}

Form Approved

OMB No. 0704-0188

Public reporting burden for the collection of information is estimated to average 1 hour per response, including the time for reviewing instructions, searching existing data sources, gathering and maintaining the data needed, and completing and reviewing the collection of information. Send comments regarding this burden estimate or any other aspect of this collection of information,

including suggestions for reducing this burden, to Washington Headquarters Services, Directorate for Information Operations and Reports, 1215 Jefferson Davis Highway, Suite 1204, Arlington

VA 22202-4302. Respondents should be aware that notwithstanding any other provision of law, no person shall be subject to a penalty for failing to comply with a collection of information if it

does not display a currently valid OMB control number.

1. REPORT DATE

2006

2. REPORT TYPE

3. DATES COVERED

00-00-2006 to 00-00-2006

4. TITLE AND SUBTITLE

Global uniqueness for a coefficient inverse problem for the non-stationary transport equation via Carleman estimate

6. $\operatorname{AUTHOR}(\mathrm{S})$

7. PERFORMING ORGANIZATION NAME(S) AND ADDRESS(ES)

a. CONTRACT NUMBER

5b. GRANT NUMBER

5c. PROGRAM ELEMENT NUMBER

5d. PROJECT NUMBER

5e. TASK NUMBER

5f. WORK UNIT NUMBER

8. PERFORMING ORGANIZATION

REPORT NUMBER

University of North Carolina at Charlotte,Department of Mathematics and Statistics,Charlotte,NC,28223

9. SPONSORING/MONITORING AGENCY NAME(S) AND ADDRESS(ES)

10. SPONSOR/MONITOR'S ACRONYM(S)

11. SPONSOR/MONITOR'S REPORT

$\operatorname{NUMBER}(\mathrm{S})$

12. DISTRIBUTION/AVAILABILITY STATEMENT

Approved for public release; distribution unlimited

13. SUPPLEMENTARY NOTES

14. ABSTRACT

15. SUBJECT TERMS

16. SECURITY CLASSIFICATION OF:

a. REPORT

unclassified

b. ABSTRACT

unclassified
17. LIMITATION OF ABSTRACT
18. NUMBER

OF PAGES

21 19a. NAME OF

RESPONSIBLE PERSON

\section{unclassified}


[13] - [15] for constructing of a globally convergent numerical method for CIPs, including the case when the initial condition is the $\delta$-function; see [19] for details and more references. A variety of works with uniqueness and stability results for coefficient inverse problems were published, which are based on the idea of [4] and [13]-[15], see, for example, Bellassoued [2], [3], Imanuvilov and Yamamoto [9], [10] and [11] for the case of hyperbolic equations. The Lipschitz stability result for the CIP for the non-stationary single-speed transport equation for $t \in(-T, T)$ with the complete lateral boundary data and initial condition at $t=0$ followed by the corresponding uniqueness result was recently obtained by the authors [18].

A natural question arises: Is it possible to consider the CIP for the non-stationary single-speed transport equation $t \in(0, T)$ rather than for $t \in(-T, T)$ and obtain at least a global uniqueness result for this case, which would be similar to [18]?

Since there are no other techniques so far, except of one of [4], [13]-[15], [19], which would enable one to prove global uniqueness for the multi-dimensional CIPs with the single measurement data, we have no choice but to use that method. However, this method doesn't necessarily allow one to consider the problem in $(0, T)$ with the initial data at $\{t=0\}$. The obstacle is due to the presence of an integral in the Carleman estimate, depending on the divergence term $V$, where the integration is carried out over $\{t=0\}$ (see Lemma 1). In the case of hyperbolic inverse problems this integral is zero due to the Carleman estimate for the principal part of the hyperbolic operator, see Theorem 2.2.4 in [19]. This is basically due to the fact that the hyperbolic operator contains the derivative of the even order (2) with respect to $t$. Thus, it is possible to consider hyperbolic inverse problems in $(0, T)$. However, in the Carleman estimates for parabolic and transport equations such integrals are not zero. Thus, these Carleman estimates do not allow to consider the corresponding inverse problems in $(0, T)$, at least directly.

Another method to try is to consider the forward problem in $(0, T)$, construct an extension of its solution into $(-T, 0)$ and consider the inverse problem simultaneously in $(0, T)$ and $(-T, 0)$. So that the integrals over $\{t=0\}$, arising from the divergent term $V$ in both cases, would cancel out. In order to use the same Carleman estimate for the problem in $(0, T)$ and $(-T, 0)$, the principal part of the differential operator should not change in such an extension.

It's quite easy to find such an extension for the hyperbolic inverse problem. For example, considering the equation

$$
u_{t t}=\Delta u+a(x) u, \quad u(x, 0)=f(x), \quad u_{t}(x, 0)=0,
$$

we see, that the extension, even with respect to $t$, leaves the form of the operator unchanged in $(-T, 0)$. However, this is not the case for parabolic problems. Indeed, considering the simple parabolic equation

$$
u_{t}=\Delta u+a(x) u, \quad u(x, 0)=f(x),
$$

and trying both even and odd extension of it's solution $u(x, t)$ into $(-T, 0)$ we see that the operator changes its form in $(-T, 0)$ due to the presence of the first derivative with respect to $t$. Thus, parabolic inverse problems are usually considered in $(-T, T)$ instead of $(0, T)$ [8], [13]-[15], [19]. Although there are some uniqueness results for parabolic inverse problems in $(0, T)$, but they are obtained via reducing a parabolic inverse problem to a hyperbolic one, using an analog of the inverse Laplace transform, see [14], [15] and subsection 3.3.1 in [19].

Our main idea is that it is possible to construct a proper extension for the non-stationary transport equation due to the presence of the parameter $v$, that represents the vector of particle velocity. To illustrate our idea consider the simplified form of the transport equation

$$
u_{t}^{+}(x, t, v)+\left(v, \nabla u^{+}(x, t, v)\right)+a(x, v) u^{+}(x, t, v)=0,
$$


in $\mathrm{R}^{n} \times(-T, 0) \times S^{n}, S^{n}=\left\{v \in \mathrm{R}^{n}:|v|=1\right\}$. Consider the following extension

$$
u^{-}(x, t, v)=u^{+}(x,-t,-v) \quad \forall(x, t, v) \in \mathrm{R}^{n} \times(-T, 0) \times S^{n} .
$$

The equation (1.1) becomes

$$
u_{t}^{-}(x, t, v)+\left(v, \nabla u^{-}(x, t, v)\right)-a(x,-v) u^{-}(x, t, v)=0, \text { in } \mathrm{R}^{n} \times(-T, 0) \times S^{n} .
$$

Hence, the principal part of the differential operator stays the same.

When trying to obtain the Lipschitz stability for the Inverse Problem formulated below, the authors have discovered that quite cumbersome conditions need to be imposed. Thus, they have decided to limit the scope of this paper to the topic of uniqueness only. In section 2 the main result (Theorem 1) is formulated, and it is proven in section 3.

\section{Statement of the main result}

Denote

$$
\begin{gathered}
\Omega=\left\{x \in \mathrm{R}^{n}:|x|<R\right\}, \quad S^{n}=\left\{v \in \mathrm{R}^{n}:|v|=1\right\}, \\
H=\Omega \times S^{n} \times(-T, T), \quad \Gamma=\partial \Omega \times S^{n} \times(-T, T), \quad Z=\Omega \times S^{n}, \\
H^{+}=\Omega \times S^{n} \times(0, T), \quad \Gamma^{+}=\partial \Omega \times S^{n} \times(0, T), \\
H^{-}=\Omega \times S^{n} \times(-T, 0), \quad \Gamma^{-}=\partial \Omega \times S^{n} \times(-T, 0),
\end{gathered}
$$

so that

$$
H=H^{+} \cup H^{-} \text {and } \Gamma=\Gamma^{+} \cup \Gamma^{-} .
$$

Also, denote

$$
\widetilde{C}^{k}(H)=\left\{s \in C^{k}(H): D_{x, t}^{\alpha} s(x, t, v) \in C(\bar{H}),|\alpha| \leq k\right\}
$$

The transport equation in the domain $\mathrm{H}^{+}$has the form [6]

$$
u_{t}+(v, \nabla u)+a(x, v) u+\int_{S^{n}} g(x, t, v, \mu) u(x, t, \mu) d \sigma_{\mu}=F(x, t, v),
$$

where $v \in S^{n}$ is a unit vector of the particle velocity, $u(x, t, v) \in \widetilde{C}^{2}\left(\overline{H^{+}}\right)$is the density of particle flow, $a(x, v)$ is an absorption coefficient, $F(x, t, v)$ is the angular density of sources, $g(x, t, v, \mu)$ is a scattering indicatrix., and $(v, \nabla u)$ denotes the scalar product of two vectors $v$ and $\nabla u$.

Consider the following boundary condition

$$
\begin{gathered}
u(x, t, v)=p(x, t, v), \\
\text { for }(x, t, v) \in\left\{(x, t, v):|x|=R, t \in(0, T), v \in S^{n}, \quad(n(x), v)<0\right\} .
\end{gathered}
$$


Here $(n(x), v)$ is the scalar product of the outer unit normal vector $n(x)$ to the surface $\partial \Omega$ and the direction of the velocity $v$. Hence, only incoming radiation is given at the boundary in this case.

Equation (2.1) with the boundary condition (2.2) and the initial condition at $t=0$

$$
u(x, 0, v)=f(x, v), \forall(x, v) \in Z,
$$

form the classical forward problem for the transport equation. Uniqueness, existence and stability results for this problem are well known, see, e. g., Prilepko and Ivankov [22].

Suppose now that the absorption coefficient $a(x, v)$ is unknown, but the following additional boundary condition is given:

$$
\begin{gathered}
u(x, t, v)=q(x, t, v), \\
\text { for }(x, t, v) \in\left\{(x, t, v):|x|=R, t \in(0, T), v \in S^{n}, \quad(n(x), v) \geq 0\right\} .
\end{gathered}
$$

The function $q(x, t, v)$ describes the outgoing radiation at the boundary.

Remark 1. When proving the uniqueness Theorem 1 for our inverse problem, we naturally assume the existence of two solutions $a_{1}(x, v)$ and $a_{2}(x, v)$ of this problem and then show that $a_{1}(x, v)=a_{2}(x, v)$. Therefore, we also assume the existence of corresponding solutions $u_{1}(x, t, v)$ and $u_{2}(x, t, v)$ of the forward problem, which, in particular satisfy the natural compatibility conditions between boundary and initial data.

Introduce the function $\gamma(x, t, v)$

$$
\gamma(x, t, v)= \begin{cases}p(x, t, v), & \text { if }(n(x), v)<0, \\ q(x, t, v), & \text { if }(n(x), v) \geq 0 .\end{cases}
$$

Hence,

$$
\left.u\right|_{\Gamma^{+}}=\gamma(x, t, v), \quad \forall(x, t, v) \in \partial \Omega \times(0, T) \times S^{n} .
$$

Thus, we obtain the following coefficient inverse problem for the non-stationary transport equation.

Inverse Problem. Given the initial condition (2.3) and the lateral boundary data (2.4), determine the coefficient $a(x, v)$ of the equation (2.1).

Theorem 1 is the main result of this paper.

Theorem 1. Suppose that derivative $\partial_{t} g$ exists in $\overline{H^{+}} \times S^{n}$ and $\left\|\partial_{t}^{k} g\right\|_{C\left(\overline{H^{+}} \times S^{n}\right)} \leq r_{1}$ for $k=0,1$, where $r_{1}$ is a positive constant. Let $|f(x, v)|>r_{2}$, where $r_{2}=$ const $>0$. Assume that there exist two pairs of functions $\left(a_{1}, u_{1}\right)$ and $\left(a_{2}, u_{2}\right)$ satisfying $(2.1),(2.3),(2.4)$ and such that

$$
a_{1}, a_{2} \in C(\bar{Z}) \text { and } u_{i}, u_{i t}, u_{i t t}, \nabla u_{i}, \nabla u_{i t} \in C\left(\overline{H^{+}}\right), i=1,2 \text {. }
$$

Suppose also that

$$
\left[\left(a_{i} f\right)(x, v)\right]^{2}=\left[\left(a_{i} f\right)(x,-v)\right]^{2}, i=1,2 .
$$

Let $\left\|u_{i t}\right\|_{C\left(\overline{H^{+}}\right)} \leq r_{3}$, where $r_{3}=$ const $>0$. Then there exists a number $T_{0}=T_{0}\left(R, r_{2}, r_{3}\right)>R$ such that if $T>T_{0}$, then $a_{1}=a_{2}$ in $Z$ and $u_{1}=u_{2}$ in $H^{+}$. If the function $u_{2}(x, t, v) \neq 0$ in $\overline{H^{+}}$, then it is sufficient to have $T>R$.

Remark 2. In particular, the condition (2.5) is satisfied if the functions $a_{i}(x, v),(i=1,2)$ and 
$f(x, v)$ satisfy one of the following pairs of relations:

$$
a_{i}(x, v)=a_{i}(x,-v) \text { and } f(x, v)=f(x,-v),
$$

or

$$
a_{i}(x, v)=a_{i}(x,-v) \text { and } f(x, v)=-f(x,-v),
$$

or

$$
a_{i}(x, v)=-a_{i}(x,-v) \text { and } f(x, v)=f(x,-v),
$$

or

$$
a_{i}(x, v)=-a_{i}(x,-v) \text { and } f(x, v)=-f(x,-v) .
$$

Remark 3. The uniqueness for the Inverse Problem considered for $t \in(-T, T)$ with initial condition given at $t=0$, though obtained as a corollary of the Lipschitz stability in [18], was obtained under stronger requirements for the differentiability of the coefficients of the transport equation (2.1), initial and boundary data, then the requirements of the Theorem 1 . Note, that the proof of the Theorem 1 is valid for the case when $t \in(-T, T)$ and the initial data is given at $t=0$, thus the assumptions of the Theorem 1, being naturally extended to the case of $t \in(-T, T)$, are sufficient for the global uniqueness of the Inverse Problem considered for $t \in(-T, T)$.

Let

$$
L_{0} u=u_{t}+(v, \nabla u)=u_{t}+\sum_{i=1}^{n} v_{i} u_{i}
$$

where $u_{i} \equiv \partial u / \partial x_{i}$. Introduce the function

$$
\psi(x, t)=|x|^{2}-\eta t^{2}, \quad \eta=\text { const } \in(0,1) .
$$

Let $c=$ const $>0$. Denote

$$
G_{c}=\left\{(x, t):|x|^{2}-\eta t^{2}>c^{2} \text { and }|x|<R\right\} .
$$

Introduce the Carleman Weight Function (CWF) as

$$
\mathrm{C}(x, t)=\exp [\lambda \psi(x, t)] .
$$

Lemma 1. Choose the number $\eta$ such that $\eta \in(0,1)$ and $T>R / \sqrt{\eta}$. Also, choose the constant $c>0$ such that $G_{c} \subset \Omega \times(-T, T)$. Then there exist positive constants $\lambda_{0}=\lambda_{0}\left(G_{c}\right)$ and $M=M\left(G_{c}\right)$, depending only on the domain $G_{c}$, such that the following pointwise Carleman estimate holds in $G_{c} \times S^{n}$ for all functions $u(x, t, v) \in C^{1}\left(\overline{G_{c}}\right) \times C\left(S^{n}\right)$ and for all $\lambda \geq \lambda_{0}\left(G_{c}\right)$

$$
\left(L_{0} u\right)^{2} C^{2} \geq 2 \lambda(1-\eta) u^{2} C^{2}+\nabla \cdot U+V_{t},
$$

where the vector function $(U, V)$ satisfies the estimate 


$$
|(U, V)| \leq M \lambda u^{2} C^{2}
$$

and

$$
V(x, t, v)=2 \lambda(\eta t-(v, x)) u^{2} \mathrm{C}^{2} .
$$

The proof of this lemma can be found in [17]. The following lemma provides an estimate from the above for an integral containing the CWF.

Lemma 2. For all functions $s \in C\left(\overline{G_{c}}\right)$ and for all $\lambda \geq 1$, the following estimate holds

$$
\int_{G_{c}}\left[\int_{0}^{t} s(x, \tau) d \tau\right]^{2} C^{2}(x, t) d x d t \leq \frac{1}{\lambda \eta} \cdot \int_{G_{c}}\left(s^{2} C^{2}\right)(x, t) d x d t .
$$

See Section 3.1 in [19] for the proof.

Lemma 3. Let $T>R$. Then for any $c \in(0, R)$ there exists a $\eta_{0}=\eta_{0}(R, T, c) \in(0,1)$ such that $G_{c} \subset \Omega \times(-T, T)$ for all $\eta \in\left(\eta_{0}(R, T, c), 1\right)$.

Proof. By the definition of the set $G_{c}$

$$
G_{c} \subset\{\Omega \times(-T, T)\} \Leftrightarrow \max _{\partial \Omega} \psi(x, T) \leq c^{2},
$$

i.e. when

$$
R^{2}-\eta T^{2} \leq c^{2}
$$

which leads to the following inequality

$$
\eta \geq \eta_{0}:=\frac{R^{2}-c^{2}}{T^{2}} .
$$

Since $c \in(0, R)$ and $R<T$ then $\eta_{0} \in(0,1)$

\section{Proof of Theorem 1}

Suppose that there exist two solutions of the Inverse Problem, $\left(a_{1}, u_{1}\right)$ and $\left(a_{2}, u_{2}\right)$. Denote

$$
\widetilde{a}=a_{1}-a_{2} \text { and } \tilde{u}=u_{1}-u_{2} .
$$

From relations (2.1), (2.3), (2.4) and (3.1), noticing that $a_{1} u_{1}-a_{2} u_{2}=a_{1} \widetilde{u}+\widetilde{a} u_{2}$, we obtain

$$
\begin{gathered}
\widetilde{u}_{t}+(v, \nabla \widetilde{u})+a_{1}(x, v) \widetilde{u}+\int_{S^{n}} g(x, t, v, \mu) \widetilde{u}(x, t, \mu) d \sigma_{\mu}=-\widetilde{a} u_{2}, \\
\widetilde{u}(x, 0, v)=0, \quad \forall(x, v) \in Z,
\end{gathered}
$$




$$
\left.\widetilde{u}\right|_{\Gamma^{+}}=0
$$

Denote

$$
\widetilde{u}^{+}(x, t, v)=\widetilde{u}(x, t, v), \quad \text { in } H^{+},
$$

Hence, (3.2)-(3.4) become

$$
\begin{gathered}
\widetilde{u}_{t}^{+}+\left(v, \nabla \widetilde{u}^{+}\right)+a_{1}(x, v) \widetilde{u}^{+}+\int_{S^{n}} g(x, t, v, \mu) \widetilde{u}^{+}(x, t, \mu) d \sigma_{\mu}=-\widetilde{a} u_{2}, \\
\widetilde{u}^{+}(x, 0, v)=0, \quad \forall(x, v) \in Z, \\
\left.\widetilde{u}^{+}\right|_{\Gamma^{+}}=0 .
\end{gathered}
$$

To apply the Carleman estimate of Lemma 1, we need to construct such an extension $\widetilde{u}^{-}(x, t, v)$ of the function $\widetilde{u}^{+}(x, t, v)$ into the domain $H^{-}$, that the principal part of the differential operator of the equation for the function $\widetilde{u}^{-}(x, t, v)$ in $H^{-}$would be the same as the one for $\widetilde{u}^{+}(x, t, v)$.

Set

$$
\widetilde{u}^{-}(x, t, v):=\widetilde{u}^{+}(x,-t,-v) \quad \forall(x, t, v) \in \bar{H}^{-} .
$$

Hence,

$$
\widetilde{u}_{t}^{-}(x, t, v)=-\widetilde{u}_{t}^{+}(x,-t,-v) \quad \forall(x, t, v) \in \overline{H^{-}} .
$$

Also, denote

$$
u_{2}^{+}(x, t, v):=u_{2}(x, t, v) \quad \forall(x, t, v) \in \overline{H^{+}}
$$

and

$$
u_{2}^{-}(x, t, v):=u_{2}(x,-t,-v) \quad \forall(x, t, v) \in \overline{H^{-}} .
$$

In the integral term in (3.2) change variables $\mu \rightarrow-\mu$ and rewrite (3.2) in the form

$$
\begin{gathered}
\widetilde{u}_{t}^{+}(x, t, v)+\left(v, \nabla \widetilde{u}^{+}(x, t, v)\right)+a_{1}(x, v) \widetilde{u}^{+}(x, t, v)+\int_{S^{n}} g(x, t, v,-\mu) \widetilde{u}^{+}(x, t,-\mu) d \sigma_{\mu} \\
=-\widetilde{a}(x, v) u_{2}(x, t, v) .
\end{gathered}
$$

Next, replace in this equation $v$ with $-v$. We obtain

$$
\begin{aligned}
\widetilde{u}_{t}^{+}(x, t,-v)-\left(v, \nabla \widetilde{u}^{+}(x, t,-v)\right)+ & a_{1}(x,-v) \widetilde{u}^{+}(x, t,-v)+\int_{S^{n}} g(x, t,-v,-\mu) \widetilde{u}^{+}(x, t,-\mu) d \sigma_{\mu} \\
& =-\widetilde{a}(x,-v) u_{2}(x, t,-v) .
\end{aligned}
$$

Now substitute $-t$ for $t$ assuming that $t<0$, 


$$
\begin{gathered}
\tilde{u}_{t}^{+}(x,-t,-v)-\left(v, \nabla \widetilde{u}^{+}(x,-t,-v)\right)+a_{1}(x,-v) \widetilde{u}^{+}(x,-t,-v)+\int_{S^{n}} g(x,-t,-v,-\mu) \widetilde{u}^{+}(x,-t,-\mu) d \sigma_{\mu} \\
=-\widetilde{a}(x,-v) u_{2}(x,-t,-v) .
\end{gathered}
$$

By $(3.7 \mathrm{a}, \mathrm{b})$ we obtain in $H^{-}$

$$
\begin{gathered}
\widetilde{u}_{t}^{-}(x, t, v)+\left(v, \nabla \widetilde{u}^{-}(x, t, v)\right)-a_{1}(x,-v) \widetilde{u}^{-}(x, t, v)- \\
-\int_{S^{n}} g(x,-t,-v,-\mu) \widetilde{u}^{-}(x, t, \mu) d \sigma_{\mu}=\widetilde{a}(x,-v) u_{2}(x,-t,-v) .
\end{gathered}
$$

Although the non-principal part of the operator for the function $\widetilde{u}^{-}$is different from the one for the function $\widetilde{u}^{+}$, but this doesn't affect the method of Carleman estimates. This is because the Carleman estimate depends only on the principal part of the differential operator. Also, by (3.6),

$$
\widetilde{u}^{-}(x, 0, v)=\widetilde{u}^{+}(x, 0,-v)=0 \quad \forall(x, v) \in \Omega \times S^{n} .
$$

Thus, we have constructed a continuous function

$$
\widehat{u}(x, t, v)=\left\{\begin{array}{lll}
\widetilde{u}^{+}(x, t, v) & \text { in } H^{+} \\
\widetilde{u}^{-}(x, t, v) & \text { in } H^{-}
\end{array}\right.
$$

Also, note that

$$
\widehat{u}(x, 0, v)=0 \quad \text { and }\left.\quad \widehat{u}\right|_{\Gamma}=0 .
$$

Since $0<r_{2} \leq|f(x, v)|$ in $Z$, there exists a small number $\varepsilon \in(0, R]$, such that

$$
u_{2}(x, t, v) \neq 0, \quad \forall(x, t, v) \in \overline{H_{\varepsilon}^{+}},
$$

where $H_{\varepsilon}^{+}=\Omega \times(0, \varepsilon) \times S^{n}$.

Thus, we can rewrite (3.5) as

$$
-\widetilde{a}=u_{2}^{-1}\left[\widetilde{u}_{t}^{+}+\left(v, \nabla \widetilde{u}^{+}\right)+a_{1}(x, v) \widetilde{u}^{+}+\int_{S^{n}} g(x, t, v, \mu) \widetilde{u}^{+}(x, t, \mu) d \sigma_{\mu}\right],
$$

in $\overline{H_{\varepsilon}^{+}}$. Since the function $\widetilde{a}(x, v)$ is independent on $t$, we can eliminate $\widetilde{a}$ from the equation (3.10) by differentiating this equation with respect to $t$. Thus, denoting

$$
k^{+}(x, t, v)=\left(\frac{u_{2 t}^{+}}{u_{2}^{+}}\right)(x, t, v)
$$

we obtain 


$$
\begin{gathered}
\widetilde{u}_{t t}^{+}+\left(v, \nabla \widetilde{u}_{t}^{+}\right)+a_{1}(x, v) \tilde{u}_{t}^{+}+\int_{S^{n}}\left(g_{t} \widetilde{u}^{+}+g_{t} \widetilde{u}_{t}^{+}\right) d \sigma_{\mu}= \\
k^{+}(x, t, v)\left[\widetilde{u}_{t}^{+}+\left(v, \nabla \widetilde{u}^{+}\right)+a_{1}(x, v) \widetilde{u}^{+}+\int_{S^{n}} g(x, t, v, \mu) \tilde{u}^{+}(x, t, \mu) d \sigma_{\mu}\right] .
\end{gathered}
$$

Denote

$$
w^{+}(x, t, v)=\widetilde{u}_{t}^{+}-k^{+} \widetilde{u}^{+}, \quad \text { in } \overline{H_{\varepsilon}^{+}} .
$$

Hence (3.12) becomes

$$
\begin{gathered}
w_{t}^{+}+\left(v, \nabla w^{+}\right)=-k_{t}^{+} \widetilde{u}^{+}-\left(v, \nabla k^{+}\right) \widetilde{u}^{+}-a_{1}(x, v) w^{+}- \\
-\int_{S^{n}} g w^{+} d \sigma_{\mu}+k^{+} \int_{S^{n}} g \widetilde{u}^{+} d \sigma_{\mu}-\int_{S^{n}} g_{t} \widetilde{u}^{+} d \sigma_{\mu}+\int_{S^{n}} g \widetilde{u}^{+} d \sigma_{\mu} .
\end{gathered}
$$

Since

$$
\widetilde{u}^{+}(x, t, v)=\int_{0}^{t} N(x, t, \tau, v) w^{+}(x, \tau, v) d \tau,
$$

where

$$
N^{+}(x, t, \tau, v)=\frac{u_{2}^{+}(x, t, v)}{u_{2}^{+}(x, \tau, v)}
$$

Turning equation (3.14) into inequality, we obtain

$$
\begin{gathered}
\left|w_{t}^{+}+\left(v, \nabla w^{+}\right)\right| \leq \\
\leq K\left[\left|w^{+}\right|+\int_{0}^{t}\left|w^{+}(x, \tau, v)\right| d \tau+\int_{S^{n}}\left|w^{+}\right| d \sigma_{\mu}+\iint_{S^{n} 0}^{t}\left|w^{+}(x, \tau, v)\right| d \tau d \sigma_{\mu}\right] .
\end{gathered}
$$

Let

$$
c^{2}=R^{2}-\eta \varepsilon^{2}
$$

consider the set $G_{c}^{+}$, where

$$
G_{c}^{+}:=G_{c} \cap\{t>0\}=\left\{|x|^{2}-\eta t^{2}>R^{2}-\eta \varepsilon^{2}\right\} \cap\{(x, t):|x|<R, t>0\},
$$

(see Fig. 1.). 


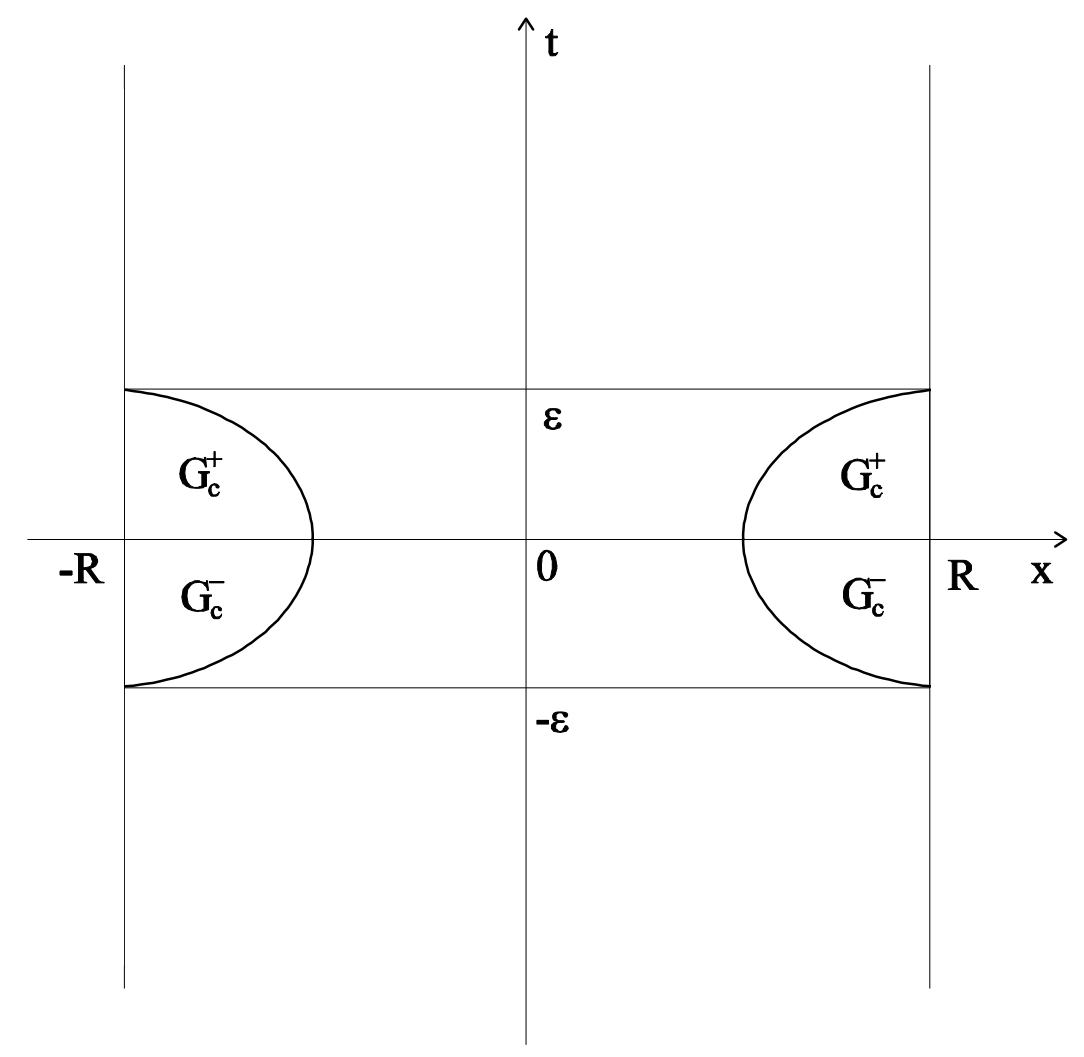

Fig. 1. 1-D schematic representation of the domains $G_{c}^{+}$and $G_{c}^{-}$.

Also. denote $P_{c}^{+}=G_{c}^{+} \times S^{n}$. Clearly,

$$
P_{c}^{+} \subset \overline{H_{\varepsilon}^{+}}
$$

Multiplying both parts of the inequality (3.16) by the CWF, squaring both sides and integrating over $P_{c}^{+}$, we obtain

$$
\begin{gathered}
\int_{P_{c}^{+}}\left|w_{t}^{+}+\left(v, \nabla w^{+}\right)\right|^{2} \mathrm{C}^{2} d h \leq \\
\leq K \int_{P_{c}^{+}}\left[w^{+}\left|+\int_{0}^{t}\right| w^{+}(x, \tau, v)\left|d \tau+\int_{S^{n}}\right| w^{+}\left|d \sigma_{\mu}+\int_{S^{n} 0}^{t}\right| w^{+}(x, \tau, v) \mid d \tau d \sigma_{\mu}\right]^{2} \mathrm{C}^{2} d h,
\end{gathered}
$$

where $d h=d x d \sigma_{v} d t$. Here and below in this proof $K$ denotes different positive constants depending on numbers $r_{1}, r_{2}, r_{3}, \varepsilon$ and norms

$$
\left\|a_{1}\right\|_{C(\bar{Z})},\left\|u_{2 t}\right\|_{C\left(\overline{H^{+}}\right)},\left\|\nabla u_{2}\right\|_{C\left(\overline{H^{+}}\right)} .
$$

We obtain 


$$
\begin{gathered}
\int w_{P_{c}^{+}}\left|w_{t}^{+}+\left(v, \nabla w^{+}\right)\right|^{2} \mathrm{C}^{2} d h \leq \\
\leq K \int_{P_{c}^{+}}\left[\left|w^{+}\right|^{2}+\left(\int_{0}^{t}\left|w^{+}(x, \tau, v)\right| d \tau\right)^{2}\right] \mathrm{C}^{2} d h+ \\
+K \int_{P_{c}^{+}}\left[\int_{S^{n}}\left|w^{+}\right|^{2} d \sigma_{\mu}+\int_{S^{n}}\left(\int_{0}^{t}\left|w^{+}(x, \tau, v)\right| d \tau\right)^{2} d \sigma_{\mu}\right] \mathrm{C}^{2} d h .
\end{gathered}
$$

Noticing that for any function $s(x, t, v) \in C\left(\overline{H^{+}}\right)$

$$
\int_{P_{c}^{+}}\left(\int_{S^{n}} s^{2} d \sigma_{\mu}\right) C^{2} d h=A \cdot \int_{P_{c}^{+}} s^{2} C^{2} d h,
$$

where $A$ is the area of the unit sphere $S^{n}$, we remove the inner integrals over $S^{n}$ in (3.18). Hence, (3.18) becomes

$$
\int_{P_{c}^{+}}\left|w_{t}^{+}+\left(v, \nabla w^{+}\right)\right|^{2} \mathrm{C}^{2} d h \leq K \int_{P_{c}^{+}}\left[\left|w^{+}\right|^{2}+\left(\int_{0}^{t}\left|w^{+}(x, \tau, v)\right| d \tau\right)^{2}\right] \mathrm{C}^{2} d h .
$$

Applying the Carleman estimate of Lemma 1, we obtain

$$
\begin{gathered}
2 \lambda(1-\eta) \int_{P_{c}^{+}}\left|w^{+}\right|^{2} \mathrm{C}^{2} d h+\int_{P_{c}^{+}}\left[\nabla \cdot U_{1}+V_{1 t}\right] d h \leq \\
\leq K \int_{P_{c}^{+}}\left|w^{+}\right|^{2} \mathrm{C}^{2} d h+K \int_{P_{c}^{+}}\left(\int_{0}^{t}\left|w^{+}(x, \tau, v)\right| d \tau\right)^{2} \mathrm{C}^{2} d h .
\end{gathered}
$$

where functions $U_{1}$ and $V_{1}$ are functions $U$ and $V$ from the Carleman estimate of Lemma 1, applied to the operator

$$
w_{t}^{+}+\left(v, \nabla w^{+}\right)
$$

Using the Gauss' formula, we obtain

$$
2 \lambda(1-\eta) \int_{P_{c}^{+}}\left|w^{+}\right|^{2} \mathrm{C}^{2} d h+\int_{\partial P_{c}^{+}}\left(\left(U_{1}, V_{1}\right), n(x, t)\right) d S \leq
$$




$$
\leq K \int_{P_{c}^{+}}\left|w^{+}\right|^{2} \mathrm{C}^{2} d h+K \int_{P_{c}^{+}}\left(\int_{0}^{t}\left|w^{+}(x, \tau, v)\right| d \tau\right)^{2} \mathrm{C}^{2} d h
$$

where $\left(\left(U_{1}, V_{1}\right), n(x)\right)$ is the scalar product of vector function $\left(U_{1}, V_{1}\right)$ and the unit outer normal $n(x, t)$ to the boundary $\partial P_{c}^{+}$. Hence, using Lemma 2 , we obtain for $\lambda>1$

$$
\begin{gathered}
2 \lambda(1-\eta) \int_{P_{c}^{+}}\left|w^{+}\right|^{2} \mathrm{C}^{2} d h+\int_{\partial P_{c}^{+}}\left(\left(U_{1}, V_{1}\right), n(x, t)\right) d S \leq \\
\leq K \int_{P_{c}^{+}}\left|w^{+}\right|^{2} \mathrm{C}^{2} d h .
\end{gathered}
$$

Choose $\lambda_{0}$ such that $K /\left(2 \lambda_{0}(1-\eta)\right)<1 / 2$. Then for all $\lambda>\max \left\{\lambda_{0}, 1\right\}$ we have from (3.19)

$$
\lambda(1-\eta) \int_{P_{c}^{+}}\left|w^{+}\right|^{2} \mathrm{C}^{2} d h+\int_{\partial P_{c}^{+}}\left(\left(U_{1}, V_{1}\right), n(x, t)\right) d S \leq 0 .
$$

Consider the boundary $\partial G_{c}^{+}$of the set $G_{c}^{+}$. Denote

$$
\begin{gathered}
\partial G_{c}^{1+}=\{(x, t):|x|=R\} \cap G_{c}^{+}, \\
\partial G_{c}^{2+}=\left\{(x, t):|x|^{2}-\eta t^{2}=c^{2}\right\} \cap G_{c}^{+}, \\
\partial G_{c}^{3+}=\{(x, t): t=0\} \cap \overline{G_{c}^{+}}
\end{gathered}
$$

Hence,

$$
\partial G_{c}^{+}=\partial G_{c}^{1+} \cup \partial G_{c}^{2+} \cup \partial G_{c}^{3+}
$$

Also, denote

$$
\partial P_{c}^{i+}=\partial G_{c}^{i+} \times S^{n}, \quad i=1,2,3 .
$$

Thus, we obtain from (3.4) and (3.20)

$$
\lambda(1-\eta) \int_{P_{c}^{+}}\left|w^{+}\right|^{2} \mathrm{C}^{2} d h+\int_{\partial P_{c}^{2+}}\left(\left(U_{1}, V_{1}\right), n(x, t)\right) d S-\int_{\partial P_{c}^{3+}} V_{1}(x, 0, v) d x d \sigma_{v} \leq 0
$$

Denote

$$
H_{\varepsilon}^{-}=\Omega \times(-\varepsilon, 0) \times S^{n}, \quad G_{c}^{-}:=G_{c} \cap\{t<0\}, \quad P_{c}^{-}=G_{c}^{-} \times S^{n} .
$$

For $c$ satisfying (3.17)

$$
P_{c}^{-} \subset \overline{H_{\varepsilon}^{-}}
$$

Also, let 


$$
\begin{gathered}
\partial G_{c}^{1-}=\{(x, t):|x|=R\} \cap \overline{G_{c}^{-}}, \\
\partial G_{c}^{2-}=\left\{(x, t):|x|^{2}-\eta t^{2}=c^{2}\right\} \cap \overline{G_{c}^{-}}, \\
\partial G_{c}^{3-}=\{(x, t): t=0\} \cap \overline{G_{c}^{-}}
\end{gathered}
$$

Hence,

$$
\partial G_{c}^{-}=\partial G_{c}^{1-} \cup \partial G_{c}^{2-} \cup \partial G_{c}^{3-}
$$

Let

$$
\partial P_{c}^{i-}=\partial G_{c}^{i-} \times S^{n}, \quad i=1,2,3 .
$$

Denote

$$
k^{-}(x, t, v)=\left(\frac{u_{2 t}^{-}}{u_{2}^{-}}\right)(x, t, v)
$$

Then

$$
\widetilde{u}^{-}(x, t, v)=\int_{0}^{t} N^{-}(x, t, \tau, v) w^{-}(x, \tau, v) d \tau
$$

where

$$
N^{-}(x, t, \tau, v)=\frac{u_{2}^{-}(x, t, v)}{u_{2}^{-}(x, \tau, v)} .
$$

Considering the equation (3.8) in the set $\overline{H_{\varepsilon}^{-}}$and using the same reasoning as above, we obtain

$$
2 \lambda(1-\eta) \int_{P_{c}^{-}}\left|w^{-}\right|^{2} \mathrm{C}^{2} d h+\int_{\partial P_{c}^{2-}}\left(\left(U_{2}, V_{2}\right), n(x, t)\right) d S+\int_{\partial P_{c}^{3-}} V_{2}(x, 0, v) d x d \sigma_{v} \leq 0,
$$

where

$$
w^{-}(x, t, v)=\widetilde{u}_{t}^{-}-k^{-} \widetilde{u}^{-}, \quad \text { in } \overline{H_{\varepsilon}^{-}} \text {. }
$$

Hence

$$
\widetilde{u}^{-}(x, t, v)=\int_{0}^{t} N^{-}(x, t, \tau, v) w^{-}(x, \tau, v) d \tau,
$$

and functions $U_{2}$ and $V_{2}$ are the functions $U$ and $V$ from the Carleman estimate of Lemma 1, applied to the operator

$$
w_{t}^{-}+\left(v, \nabla w^{-}\right)
$$

Consider the set $P_{c}=P_{c}^{-} \cup P_{c}^{+}$. By (3.21) and (3.23) 


$$
\partial P_{c}^{3-}=\partial P_{c}^{3+}=\{x: c<|x|<R\} \times S^{n}:=\partial P_{c}^{3} .
$$

Hence, summing up the inequalities (3.22) and (3.24), we obtain

$$
\begin{gathered}
\lambda(1-\eta) \int_{P_{c}}|\widehat{w}|^{2} \mathrm{C}^{2} d h+\int_{\partial P_{c}^{2+}}\left(\left(U_{1}, V_{1}\right), n(x, t)\right) d S+\int_{\partial P_{c}^{2-}}\left(\left(U_{2}, V_{2}\right), n(x, t)\right) d S+ \\
+\int_{\partial P_{c}^{3}}\left[V_{2}(x, 0, v)-V_{1}(x, 0, v)\right] d x d \sigma_{v} \leq 0 .
\end{gathered}
$$

Here the function $\widehat{w}$ is defined as

$$
\widehat{w}(x, t, v)= \begin{cases}w^{+}(x, t, v) & \text { in } H^{+} \\ w^{-}(x, t, v) & \text { in } H^{-} .\end{cases}
$$

We first need to cancel out the possibly non-zero integral

$$
\int_{\partial P_{c}^{3}}\left[V_{2}(x, 0, v)-V_{1}(x, 0, v)\right] d x d \sigma_{v} .
$$

Actually, this is the central point of the proof compared with the case of considering $(-T, T)$ instead of $(0, T)$ (see Introduction). By (2.7)

$$
V_{1}(x, 0, v)=-2 \lambda(v, x)\left(w^{+}(x, 0, v)\right)^{2} \mathrm{C}^{2},
$$

and

$$
V_{2}(x, 0, v)=-2 \lambda(v, x)\left(w^{-}(x, 0, v)\right)^{2} \mathrm{C}^{2} .
$$

Hence, if

$$
\left[\left(w^{+}(x, 0, v)\right]^{2}=\left[w^{-}(x, 0, v)\right]^{2},\right.
$$

then

$$
V_{2}(x, 0, v)-V_{1}(x, 0, v)=0 \text { in } \partial P_{c}^{3} .
$$

By (3.9), (3.13) and (3.25)

$$
w^{+}(x, 0, v)=\widetilde{u}_{t}^{+}(x, 0, v) \text { and } w^{-}(x, 0, v)=\widetilde{u}_{t}^{-}(x, 0, v),
$$

and by (2.3), (3.5) and (3.8)

$$
w^{+}(x, 0, v)=-\widetilde{a}(x, v) u_{2}(x, 0, v)=-\widetilde{a}(x, v) f(x, v),
$$

and 


$$
w^{-}(x, 0, v)=\widetilde{a}(x,-v) u_{2}(x, 0,-v)=\widetilde{a}(x,-v) f(x,-v)
$$

for all $(x, v) \in P_{c}^{3}$. Thus (3.29) is equivalent to

$$
[\widetilde{a}(x, v) f(x, v)]^{2}=[\widetilde{a}(x,-v) f(x,-v)]^{2}, \quad \forall(x, v) \in P_{c}^{3} .
$$

Because of (3.30), the integral (3.28) equals zero and the inequality (3.27) becomes

$$
\begin{gathered}
\lambda(1-\eta) \int_{P_{c}}|\widehat{w}|^{2} \mathrm{C}^{2} d h+\int_{\partial P_{c}^{2+}}\left(\left(U_{1}, V_{1}\right), n(x, t)\right) d S+ \\
+\int_{\partial P_{c}^{2-}}\left(\left(U_{2}, V_{2}\right), n(x, t)\right) d S \leq 0 .
\end{gathered}
$$

The inequality (3.31) leads to

$$
\begin{gathered}
2 \lambda(1-\eta) \int_{P_{c}}|\widehat{w}|^{2} \mathrm{C}^{2} d h \leq \int_{\partial P_{c}^{2+}}\left|\left(U_{1}, V_{1}\right)\right| d S+ \\
\quad+\int_{\partial P_{c}^{2-}}\left|\left(U_{2}, V_{2}\right)\right| d S .
\end{gathered}
$$

Denote $\partial P_{c}^{2}=\partial P_{c}^{2-} \cup \partial P_{c}^{2+}$. Hence, using the estimate (2.6), we obtain from (3.32)

$$
2 \lambda(1-\eta) \int_{P_{c}}|\widehat{w}|^{2} \mathrm{C}^{2} d h \leq K \lambda \int_{\partial P_{c}^{2}}|\widehat{w}|^{2} \mathrm{C}^{2} d S .
$$

Let $\delta \in(0, \varepsilon)$ be an arbitrary number. Denote

$$
G_{c \delta}=\left\{|x|^{2}-\eta t^{2}>R^{2}-\eta \delta^{2}\right\} \cap\{|x|<R\},
$$

and $P_{c \delta}=G_{c \delta} \times S^{n}$ (see Fig. 2). 


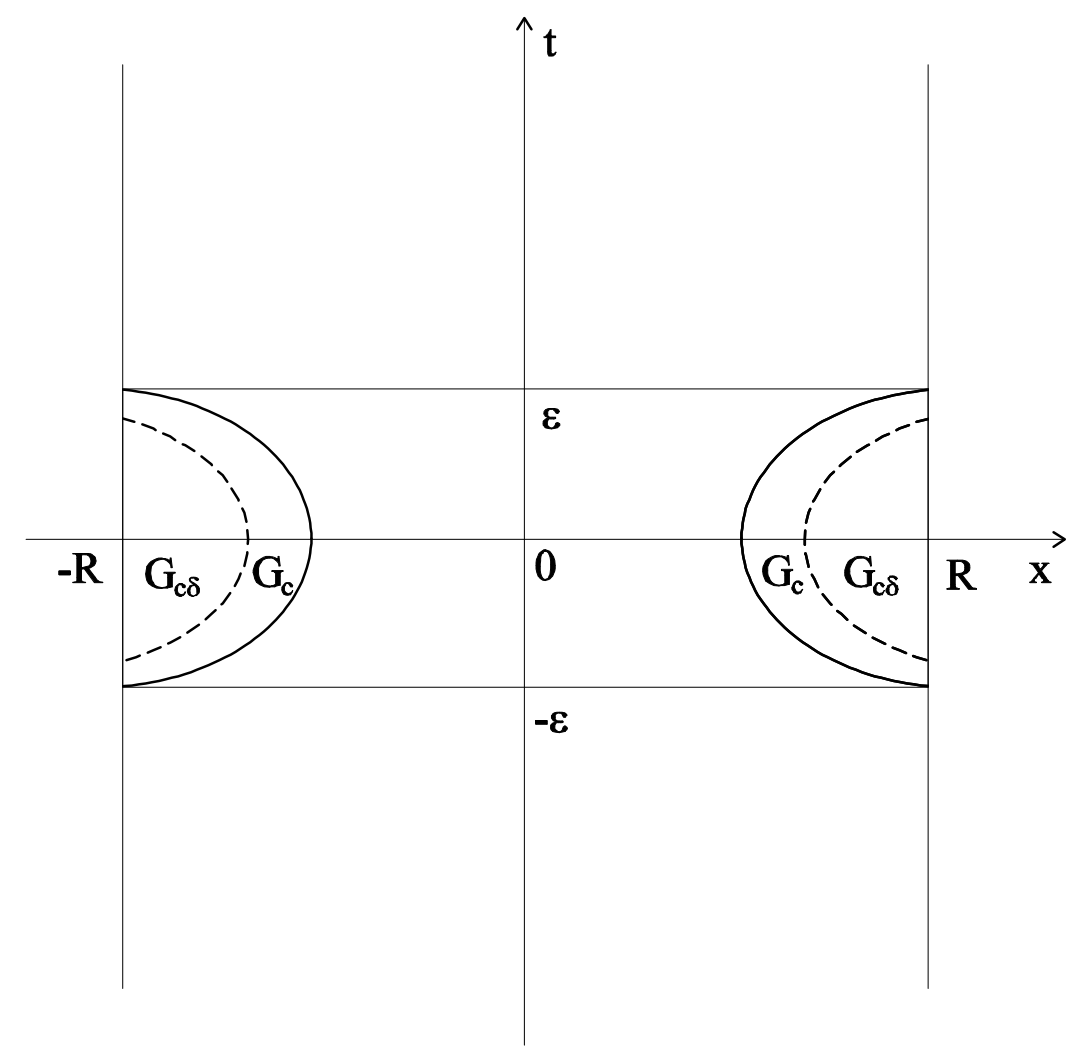

Fig. 2. Schematic representation of the sets $G_{c}$ and $G_{c \delta}$ in the 1-D case.

By (3.17) $P_{c \delta} \subset P_{c}$. Hence,

$$
\int_{P_{c \delta}}|\widehat{w}|^{2} \mathrm{C}^{2} d h \leq \int_{P_{c}}|\widehat{w}|^{2} \mathrm{C}^{2} d h
$$

Since

$$
\mathrm{C}^{2}(x, t)>\exp \left[2 \lambda\left(R^{2}-\eta \delta^{2}\right)\right] \text { in } P_{c \delta}
$$

and

$$
\mathrm{C}^{2}(x, t)=\exp \left[2 \lambda\left(R^{2}-\eta \varepsilon^{2}\right)\right] \text { on } \partial P_{c}^{2},
$$

we obtain from (3.33) and (3.34)

$$
\exp \left[2 \lambda\left(R^{2}-\eta \delta^{2}\right)\right] \int_{P_{c \delta}}|\widehat{w}|^{2} d h \leq \operatorname{Kexp}\left[2 \lambda\left(R^{2}-\eta \varepsilon^{2}\right)\right] \int_{\partial P_{c}^{2}}|\widehat{w}|^{2} d S .
$$

Dividing this inequality by $\exp \left[2 \lambda\left(R^{2}-\eta \delta^{2}\right)\right]$ and letting $\lambda \rightarrow \infty$, we obtain

$$
\int_{P_{c \delta}}|\widehat{w}|^{2} d h \leq 0,
$$

and thus 


$$
\widehat{w}(x, t, v)=0 \text { in } P_{c \delta} .
$$

Since $\delta \in(0, \varepsilon)$ is arbitrary number, then

$$
\widehat{w}(x, t, v)=0 \text { in } P_{c} .
$$

Thus, by (3.15) and (3.26) we have

$$
\widehat{u}(x, t, v)=0 \text { in } P_{c} .
$$

Since $\eta \in(0,1)$ is an arbitrary number, then (3.10), (3.35) and (3.36) imply that

$$
\widetilde{a}(x, v)=0 \text { in }\left\{\sqrt{R^{2}-\varepsilon^{2}}<|x|<R\right\} \times S^{n} .
$$

Let $t_{0} \in(-T, T)$ be a number which we will choose later. Denote

$$
G_{\varepsilon}\left(t_{0}\right):=\left\{(x, t):|x|^{2}-\eta\left(t-t_{0}\right)^{2}>R^{2}-\eta \varepsilon^{2},|x|<R\right\} .
$$

We need to have $G_{\varepsilon}\left(t_{0}\right) \subset\{t:|t|<T\} \times \Omega$. Since

$$
t_{0}-\varepsilon<t<t_{0}+\varepsilon \text { in } G_{\varepsilon}\left(t_{0}\right),
$$

we choose $t_{0} \in(-T+\varepsilon, T-\varepsilon)$. Also, denote $P_{c}\left(t_{0}\right):=G_{c}\left(t_{0}\right) \times S^{n}$ (see Fig. 3).

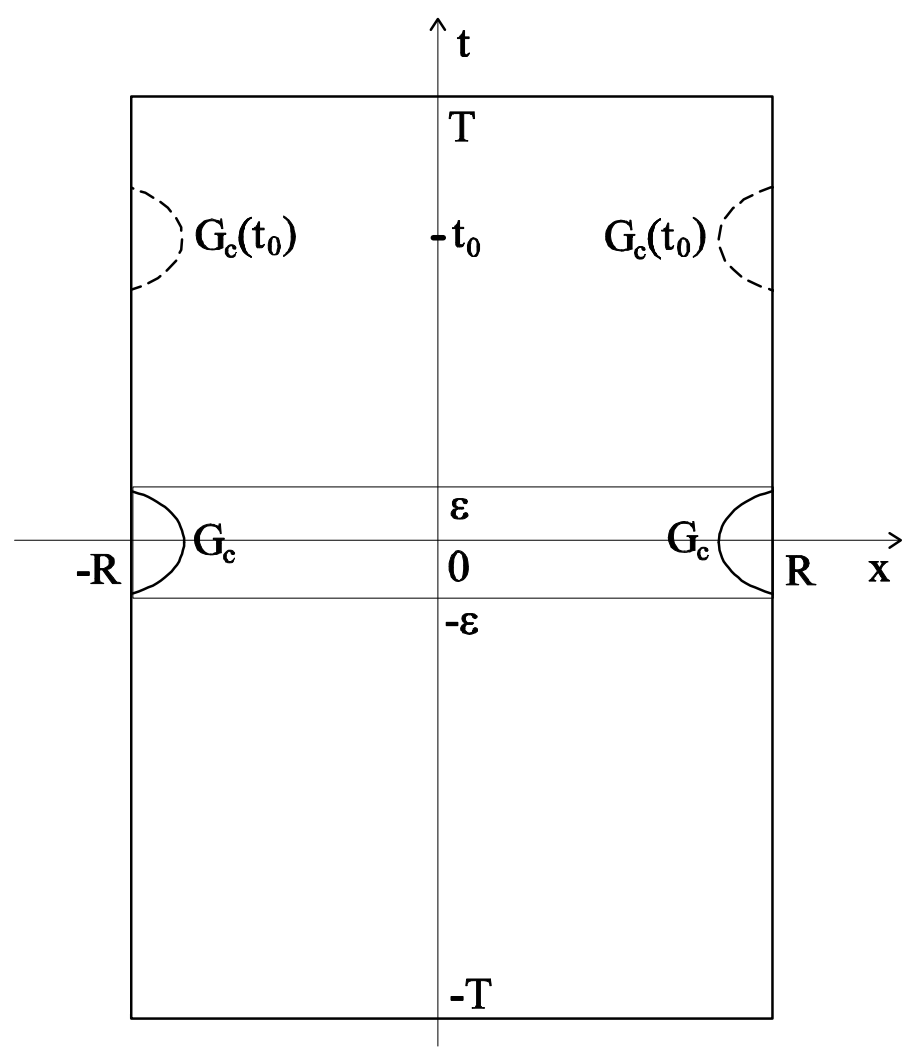

Fig. 3. Schematic representation of the sets $G_{c}$ and $G_{c}\left(t_{0}\right)$ in 1-D case.

By (3.37), the equations (3.5) and (3.8) in the domain $P_{c}\left(t_{0}\right)$ become respectively 


$$
\widetilde{u}_{t}^{+}+\left(v, \nabla \widetilde{u}^{+}\right)+a_{1}(x, v) \widetilde{u}^{+}+\int_{S^{n}} g(x, t, v, \mu) \widetilde{u}^{+}(x, t, \mu) d \sigma_{\mu}=0,
$$

for $t>0$, and

$$
\begin{gathered}
\widetilde{u}_{t}^{-}(x, t, v)+\left(v, \nabla \widetilde{u}^{-}(x, t, v)\right)-a_{1}(x,-v) \widetilde{u}^{-}(x, t, v)- \\
-\int_{S^{n}} g(x,-t,-v,-\mu) \widetilde{u}^{-}(x, t, \mu) d \sigma_{\mu}=0,
\end{gathered}
$$

for $t<0$. Since these are the homogeneous equations, we do not need to divide by $u_{2}(x, t, v)$ (see (3.11)) to prove that the conditions (3.6) and (3.7) imply that their solutions are zeros. In other words, using the above method but without the introduction of the functions $w^{+}$and $w^{-}$, we establish that

$$
\widetilde{u}^{+}(x, t, v)=0 \text { in } P_{c}\left(t_{0}\right) \cap\{t>0\}
$$

and

$$
\widetilde{u}^{-}(x, t, v)=0 \text { in } P_{c}\left(t_{0}\right) \cap\{t<0\} .
$$

Therefore,

$$
\widehat{u}(x, t, v)=0 \text { in }\left\{(x, t): \sqrt{R^{2}-\varepsilon^{2}}<|x|<R, t \in(-T+\varepsilon, T-\varepsilon)\right\} \times S^{n} .
$$

This means that now we can consider equations (3.5) and (3.8) in the domains

$$
\left\{(x, t):|x|<\sqrt{R^{2}-\varepsilon^{2}}, t \in(0, T-\varepsilon)\right\} \times S^{n},
$$

and

$$
\left\{(x, t):|x|<\sqrt{R^{2}-\varepsilon^{2}}, t \in(-T+\varepsilon, 0)\right\} \times S^{n},
$$

respectively, (see Fig. 4), and the boundary $\Gamma$ of the domain $H$ should be replaced with

$$
\left\{|x|=\sqrt{R^{2}-\varepsilon^{2}}, t \in(0, T-\varepsilon)\right\} \times S^{n} .
$$




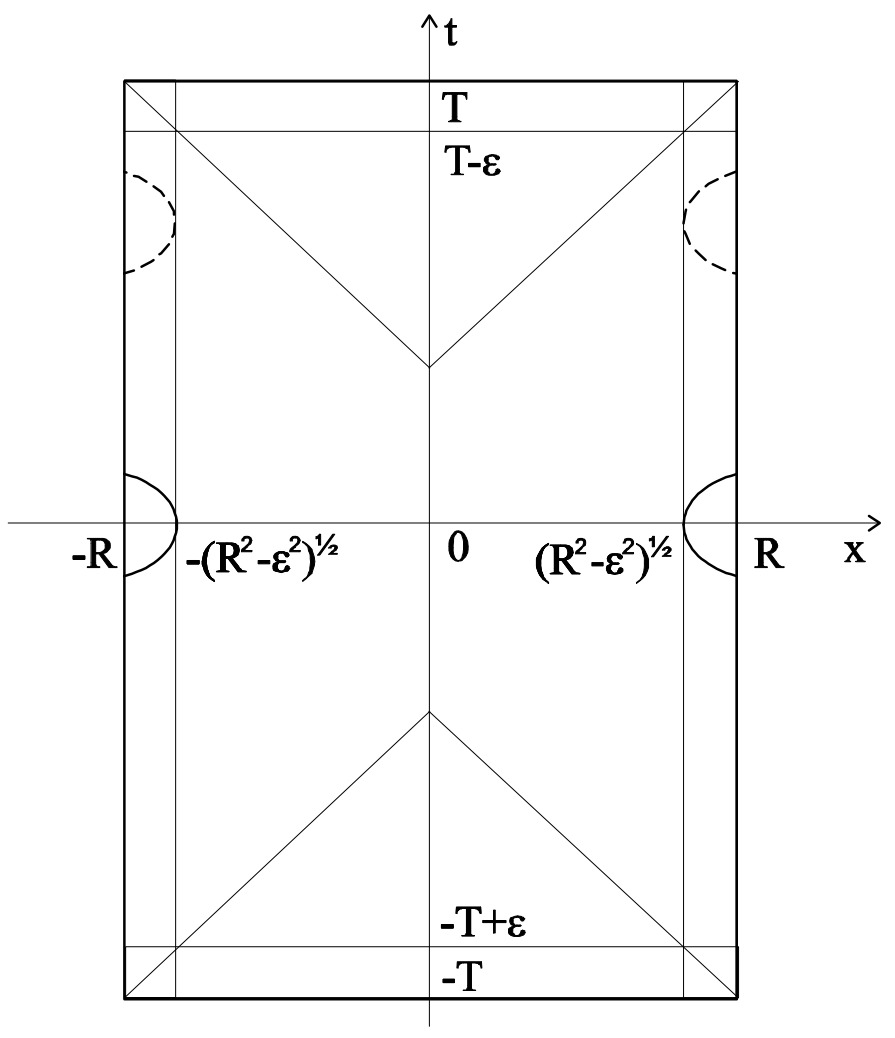

Fig. 4. Schematic representation of the new domains for 1-D case.

Repeating the above proof in this new domain, we conclude that

$$
\widetilde{a}(x, v)=0 \text { in }\left\{\sqrt{R^{2}-2 \varepsilon^{2}}<|x|<R\right\} \times S^{n} .
$$

Without the loss of generality we can assume that $R^{2}=m \varepsilon^{2}$, where $m$ is an integer. Repeating the above process $m$ times, we obtain

$$
\widetilde{a}(x, v)=0 \text { in } Z \text {. }
$$

However, to make sure that we indeed can repeat this process $m$ times, we need to have the observation interval $(0, T)$ to be sufficiently large. We now establish an estimate from below for the number $T$. We need to have $u_{2}(x, t, v) \neq 0$ in $\overline{H_{\varepsilon}^{+}}$. We have

$$
u_{2}(x, t, v)=f(x, v)+\int_{0}^{t} u_{2 t}(x, \tau, v) d \tau \text {. }
$$

Hence, $\left|u_{2}(x, t, v)\right| \geq r_{2}-t r_{3}$ in $H^{+}$. In order to have $r_{2}-t r_{3}>0$, we need to take $t>r_{2} / r_{3}$. Hence, we choose

$$
0<\varepsilon<\min \left(\frac{r_{2}}{2 r_{3}}, R\right) .
$$


To repeat that process $m$ times, we should have

$$
T>m \varepsilon=\frac{R^{2}}{\varepsilon}>\frac{R^{2}}{\min \left(\frac{r_{2}}{2 r_{3}}, R\right)},
$$

which gives us an estimate for the number $T$ from the below. In particular, if

$$
\min \left(\frac{r_{2}}{2 r_{3}}, R\right)=R
$$

then we can choose $\varepsilon=R$ and the estimate (3.39) yields $T>R$.

Finally, to prove that $\widetilde{u}^{+}(x, t, v)=0$ in $H^{+}$, we observe that because of (3.38) the equation (3.5) becomes

$$
\widetilde{u}_{t}^{+}+\left(v, \nabla \widetilde{u}^{+}\right)+a_{1}(x, v) \widetilde{u}^{+}+\int_{S^{n}} g(x, t, v, \mu) \widetilde{u}^{+}(x, t, \mu) d \sigma_{\mu}=0 .
$$

Using the standard method of energy estimates with the initial condition (3.6) and the zero boundary condition $\left.\widetilde{u}^{+}\right|_{\Gamma^{+}}=0$, we obtain

$$
\widetilde{u}^{+}(x, t, v)=0 \text { in } H^{+} .
$$

\section{Acknowledgment}

The work of M.V. Klibanov was supported by the U.S. Army Research Laboratory and U.S. Army Research Office under contract/grant number W911NF-05-1-0378.

\section{References}

[1] D. S. ANIKONOV, A. E. KOVTANYUK and I. V. PROKHOROV, Transport Equation and Tomography, VSP Publ., Utrecht, The Netherlands, 2002.

[2] M. BELLASSOUED, Global logarithmic stability for inverse hyperbolic problem by arbitrary boundary observation, Inverse Problems, 20(2004), pp. 1033-1052.

[3] M. BELLASSOUED, Uniqueness and stability in determining the speed of propagation of second-order hyperbolic equation with variable coefficients, Applicable Analysis, 83(2004), pp. 983-1014.

[4] A. L. BUKHGEIM and M. V. KLIBANOV, Uniqueness in the large of a class of multidimensional inverse problems, Soviet Math. Doklady, 17(1981), pp. 244-247.

[5] T. CARLEMAN, Sur un probléme dunicité pour les systémes d'equations aux d'erivées partielles à deux variables indépendantes, Ark. Mat. Astr. Fys., 26B, 17(1939), pp. 1-9.

[6] K. M. CASE and P. F. ZWEIFEL, Linear Transport Theory, Addison-Wesley, Reading, MA, 1967.

[7] L. HÖRMANDER, Linear Partial Differential Operators, Springer-Verlag, Berlin, 1963.

[8] O. Y. IMANUVILOV and M. YAMAMOTO, Lipschitz stability in inverse parabolic problems by the Carleman estimate, Inverse Problems 14(1998), 5, 1229-1245.

[9] O. Y. IMANUVILOV and M. YAMAMOTO, Global Lipschitz stability in an inverse hyperbolic problem by interior observations, Inverse Problems, 17(2001), pp. 717-728.

[10] O. Y. IMANUVILOV and M. YAMAMOTO, Global uniqueness and stability in determining coefficients of wave equations, Commun. in Part. Diff. Eqs., 26(2001), pp. 1409-1425. 
[11] O. Y. IMANUVILOV and M. YAMAMOTO, Determination of a coefficient in an acoustic equation with a single measurement, Inverse Problems, 19(2003), pp. 157-171.

[12] M. KAZEMI and M. V. KLIBANOV, Stability estimates for ill-posed Cauchy problem involving hyperbolic equations and inequalities, Applicable Analysis, 50(1993), pp. 93-102.

[13] M. V. KLIBANOV, Uniqueness in the large of some multidimensional inverse problems, in Non-Classical Problems of Mathematical Physics, Proc. Computing Center of the Siberian Branch of the Russian Academy of Science, Novosibrisk, 1981, pp. 101-114. (In Russian). (Also available online at http://www.math.uncc.edu/people/research/mklibanv.php3).

[14] M. V. KLIBANOV, Inverse problems in the "large and Carleman bounds, Differential Equations, 20(1984), pp. 755-760. (Also available online at http://www.math.uncc.edu/people/research/mklibanv.php3).

[15] M. V. KLIBANOV, Inverse problems and Carleman estimates, Inverse Problems, 8(1992) pp. 575-596.

[16] M. V. KLIBANOV and J. MALINSKY, Newton-Kantorovich method for 3-dimensional potential inverse scattering problem and stability of the hyperbolic Cauchy problem with time dependent data, Inverse Problems, 7(1991), pp. 577-595.

[17] M. V. KLIBANOV and S. E. PAMYATNYKH, Lipschitz stability of a non-standard problem for the non-stationary transport equation via Carleman estimate, Inverse Problems, 22(2006), pp. 881-890.

[18] M. V. KLIBANOV and S. E. PAMYATNYKH, Lipschitz stability for a coefficient inverse problem for the non-stationary transport equation via Carleman estimate, Http://www.ma.utexas.edu/mp_arc/, preprint number 06-94.

[19] M. V. KLIBANOV and A. TIMONOV, Carleman Estimates for Coeffcient Inverse Problems and Numerical Applications, VSP Publ., Utrecht, The Netherlands, 2004.

[20] M. V. KLIBANOV and A. TIMONOV, Global uniqueness for a $3 d / 2 d$ inverse conductivity problem via the modified method of Carleman estimates, J. Inverse and Ill-Posed Problems, 13(2005), pp. 149-174.

[21] M. M. LAVRENT'EV, V. G. ROMANOV and S. P. SHISHATSKII, Ill-posed problems of mathematical physics and analysis, AMS, Providence, Rhode Island, 1986.

[22] A. I. PRILEPKO and A. L. IVANKOV, Inverse problems for non-stationary transport equation, Soviet Math. Doklady, 29(1984), 3, pp. 559-564.

[23] A. I. PRILEPKO and A. L. IVANKOV, Inverse problems for the determination of a coefficient and the right side of a non-stationary multivelocity transport equation with overdetermination at a point, Differential equations, 21(1985), 1, pp. 109-119.

[24] A. I. PRILEPKO and A. L. IVANKOV, Inverse problems of finding a coefficient, the scattering indicatrix, and the right side of a nonstationary many-velocity transport equation, Differential equations, 21(1985), 5, pp. 870-885.

[25] V. G. ROMANOV, Investigation Methods for Inverse Problems, VSP Publ., Utrecht, The Netherlands, 2002.

[26] P. STEFANOV, Inverse problems in transport theory, in Inside out: inverse problems and applications, Math. Sci. Res. Inst. Publ., 47, Cambridge Univ. Press, Cambridge, 2003, pp.111-131.

[27] A. TAMASAN, An inverse boundary value problem in two-dimensional transport, Inverse Problems, 18(2002), 1, pp. 209-219. 